\title{
Influence of the diet on the composition of faecal bile acids in rats*
}

\author{
By B. E. GUSTAFSSON \\ Department of Germfree Research, Karolinska Institutet, Stockholm, Sweden \\ AND A. NORMAN \\ Department of Clinical Chemistry, Danderyds Sjukhus, Danderyd, Sweden
}

(Received 6 December 1968-Accepted ${ }_{3} 3$ February 1969)

\begin{abstract}
1. $\left[24^{-14} \mathrm{C}\right]$ cholic acid was given orally to rats receiving three different diets, a semisynthetic diet $\left(\mathrm{D}_{7}\right)$ with and without $20 \%$ cellulose, and a commercial, pelleted diet. The labelled metabolites excreted in the faeces in the 8 days following administration of the labelled cholic acid were chromatographically separated, and tentatively identified by means of thin-layer chromatography.

2. In all three dietary groups the faecal bile acids mainly consisted of unconjugated and $7 \alpha$-dehydroxylated metabolites. In rats receiving pellets or diet $D_{7}$ with cellulcse, there was a higher percentage of monohydroxy-monoketo-cholanoic acids than in those receiving diet $\mathrm{D}_{7}$ alone. Dihydroxy-cholanoic acids, present in approximately the same ratio as were the $3 \alpha-$ and $3 \beta$-hydroxy isomers, were excreted by all the rats in the three dietary groups.
\end{abstract}

The primary bile acids, cholic and chenodeoxycholic acid, are transformed during enterohepatic circulation into secondary bile acids, which are formed initially by the action of intestinal micro-organisms, and are partly absorbed, but which can be further modified in the liver, and partly excreted in the faeces. After isolating and identifying the faecal bile acids from man, Eneroth, Gordon, Ryhage \& Sjövall ( 1966 ) showed that these acids form a very complicated mixture of various different metabolites. In addition to the hydrolysis of conjugates, the main transformations of the resulting unconjugated bile acids, that take place in the intestinal tract, are: elimination of the hydroxyl groups at C-7; oxidation of hydroxyl groups at $\mathrm{C}-3, \mathrm{C}-7$, and $\mathrm{C}-\mathrm{I} 2$ to keto groups which can subsequently be reduced to both $\alpha$ - and $\beta$-hydroxyl groups (for a review see Danielsson, 1963 ).

The transformations of both conjugated and unconjugated bile acids have been studied in vitro in cultures of some microbial strains isolated from the intestinal contents of man and different animal species (Midtvedt $\&$ Norman, 1967). It was shown that the hydrolysis of conjugates is a common property of intestinal micro-organisms. The hydroxyl groups at C-3 and C-7 were oxidized by several strains, and those at $\mathrm{C}-\mathrm{I} 2$ by a few of the strains examined. Reduction of the keto groups at $\mathrm{C}-3$ has yielded both $\alpha$ - and $\beta$-hydroxyl groups, but reduction of the keto group at $\mathrm{C}-7$ has, as yet, only yielded the $7 \alpha$-hydroxyl group. Strains capable of $7 \alpha$-dehydroxylation have been isolated from the intestinal contents of rat and man (Gustafsson, Midtvedt \& Norman, I966). In the blind-loop syndrome, an increased hydrolysis of conjugated bile acids

* This work is part of investigations supported by the Swedish Medical Research Council grants 205,206 and 602 . 
takes place in the small intestine (Dawson, 1967 ). Several strains of intestinal microorganisms capable of hydrolysing conjugates have been isolated from aspirates of the small intestine of patients with this disease (Draser, Hill \& Shiner, I966; Tabaqchali \& Booth, 1966).

It is well known that the diet influences the composition of the gastro-intestinal microflora (for references see Gall, Fenton \& Cowgill, r948). As the 'normal' flora contains strains that differ in their abilities to transform bile acids, it is obvious that the diet may influence the composition of the faecal bile acids through changes in the gastro-intestinal microflora.

The aim of the present investigation was to compare the faecal metabolites of $\left[24^{-14} \mathrm{C}\right] \mathrm{cholic}$ acid excreted by rats on three different diets; a semi-synthetic diet $\left(\mathrm{D}_{7}\right)$, the same diet with $20 \%$ cellulose ( $\mathrm{D}_{7}$ with cellulose), and a commercial diet (pellets). This investigation formed part of studies using these diets, on the rate of elimination of $\left[24^{-14} \mathrm{C}\right]$ cholic acid by germ-free and conventional rats (Gustafsson \& Norman, 1969), and on the physical state of the labelled bile acids in the intestinal contents of these rats (Gustafsson \& Norman, 1968).

\section{EXPERIMENTAL AND RESULTS}

The following systematic names are given to compounds referred to in this report by trivial names: cholic acid, $3 \alpha, 7 \alpha, \mathrm{r} 2 \alpha$-trihydroxy- $5 \beta$-cholanoic acid; chenodeoxycholic acid, $3 \alpha, 7 \alpha$-dihydroxy- $5 \beta$-cholanoic acid; deoxycholic acid, $3 \alpha$, 12 $\alpha$-dihydroxy- $5 \beta$ cholanoic acid; lithocholic acid, $3 \alpha$-monohydroxy- $\beta \beta$-cholanoic acid.

\section{Diet and treatment of animals}

The experiments to be reported were carried out at the same time; on the same dietary groups of conventional rats, as those described in a previous paper (Gustafsson \& Norman, 1969), where the composition of the diets, housing of the animals and the methods of sampling of faeces and urine are described. Each rat was given $0.1 \mathrm{mg}$ of sodium $\left[24^{-14} \mathrm{C}\right]$ cholate $(5 \mu \mathrm{c})$ orally. Faeces were collected daily during 8 days from animals in which coprophagy was prevented by restraining cages (Bollman, Cain \& Grindlay, 1948), and also from animals in which coprophagy was not prevented.

\section{Chromatographic technique}

Partition column chromatography was used, as described earlier (Norman, I964), to obtain group separations of $\left[24^{-14} \mathrm{C}\right]$ cholic acid metabolites. After chromatography with phase system F I (Fig. I), cholic and 3,I2-dihydroxy-7-keto-5 $\beta$-cholanoic acid appear with, or just after, the front, and these two acids are separated from deoxycholic acid, 3 -hydroxy-I 2 -keto- $5 \beta$-cholanoic acid and 12 -hydroxy- 3 -keto- $5 \beta$-cholanoic acid. The last three acids appear in the $40-60 \mathrm{ml}$, and $80-110 \mathrm{ml}$ effluent fractions, respectively, while 3,12 -diketo- $5 \beta$-cholanoic acid is retained in the stationary phase. However, the $\alpha$ - and $\beta$-hydroxy isomers of all these acids cannot be separated by this method. This separation was achieved as follows: the bile acids were methylated and fractionated on columns of aluminium oxide (grade III; Woelm, Schwege, Germany) with a solvent system consisting of increasing concentrations of ethyl acetate in 
benzene (Danielsson, Eneroth, Hellström, Lindstedt \& Sjövall, 1963). The labelled material isolated in this way was identified by thin-layer chromatography (TLC) in different phase systems (Eneroth, 1963), together with appropriate standards. After TLC, the radioactive spots were located by autoradiography. The steps used for the separation of the various faecal metabolites are outlined in Fig. I.

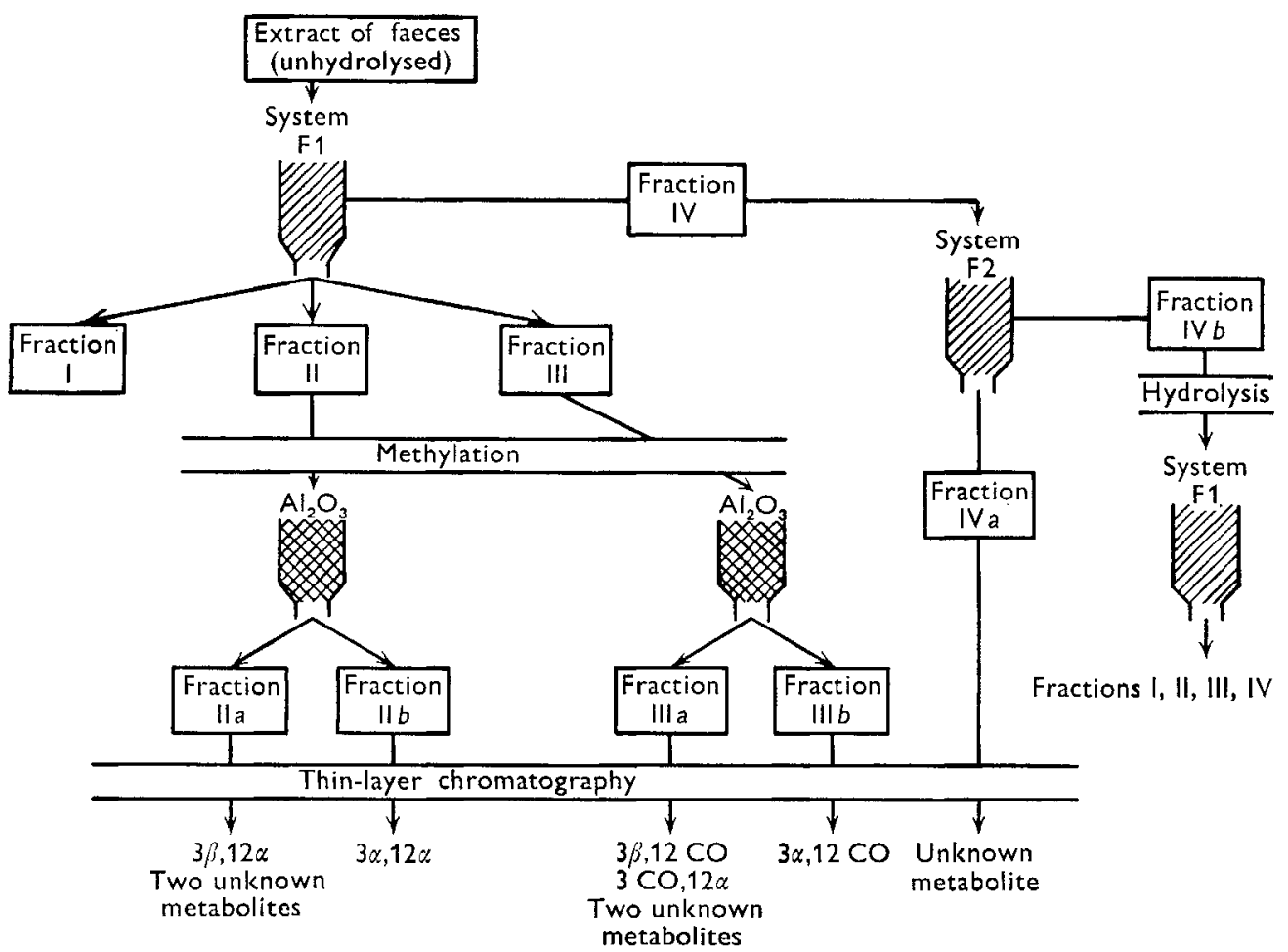

Fig. I. Outline for the fractionation and separation of metabolites of cholic acid in faeces. The following abbreviations are used: $3 \beta, \mathrm{I} 2 \alpha, 3 \beta$, 1 $2 \alpha$-dihydroxy-5 $\beta$-cholanoic acid; $3^{\alpha, 12 \alpha}$, $3 \alpha$, I $2 \alpha$-dihydroxy-5 $\beta$-cholanoic acid; $3 \beta$, 12 $\mathrm{CO}, 3 \beta$-hydroxy-1 2-keto-5 $\beta$-cholanoic acid; ${ }_{3} \mathrm{CO}, \quad$ I2 $\alpha$, I $2 \alpha$-hydroxy-3-keto- $5 \beta$-cholanoic acid; $3 \alpha, 12 \mathrm{CO}, \quad 3 \alpha$-hydroxy-12-keto-5 $\beta$ cholanoic acid.

\section{Extraction of faecal bile acids}

The faeces were collected separately for each day from individual rats for 8 days after the administration of $\left[24^{-14} \mathrm{C}\right]$ cholic acid, and stored at $-17^{\circ}$ until they were analysed. Each of these daily samples was then homogenized in water, ethanol was added to give a final concentration of $80 \%$, and the mixture was boiled for $12 \mathrm{~h}$ and filtered. Equal portions of the filtrates obtained from each of these samples were combined, evaporated, dissolved in water, acidified to $\mathrm{pH}$ I with hydrochloric acid, and then extracted with dicthyl ether followed by butanol. The labelled material in the faeces was separated into two groups by the ether extraction. Unconjugated bile acids and glycine conjugates of mono- and dihydroxy-cholanoic acids were recovered in the ether extracts, whereas glycinc conjugates of trihydroxy-cholanoic acids, and all taurine conjugates, appeared in the subsequent butanol extracts. The labelled material 
in the butanol extract did not exceed $2 \%$ of the total recovered isotope in any of the experiments. Thus a maximum of $2 \%$ cholic acid was excreted in faeces either as taurine conjugates or glycocholic acid.

\section{Chromatographic separation of labelled faecal metabolites of $\left[24^{-14} \mathrm{C}\right]$ cholic acid}

The ether extracts were chromatographed with phase system $F_{I}$, and a representative chromatogram is shown in Fig. 2. Labelled material representing four bile acid regions, three in the eluate and one retained in the column, was obtained. This was further separated as shown in Fig. I, and the following fractions were recognized.

A B

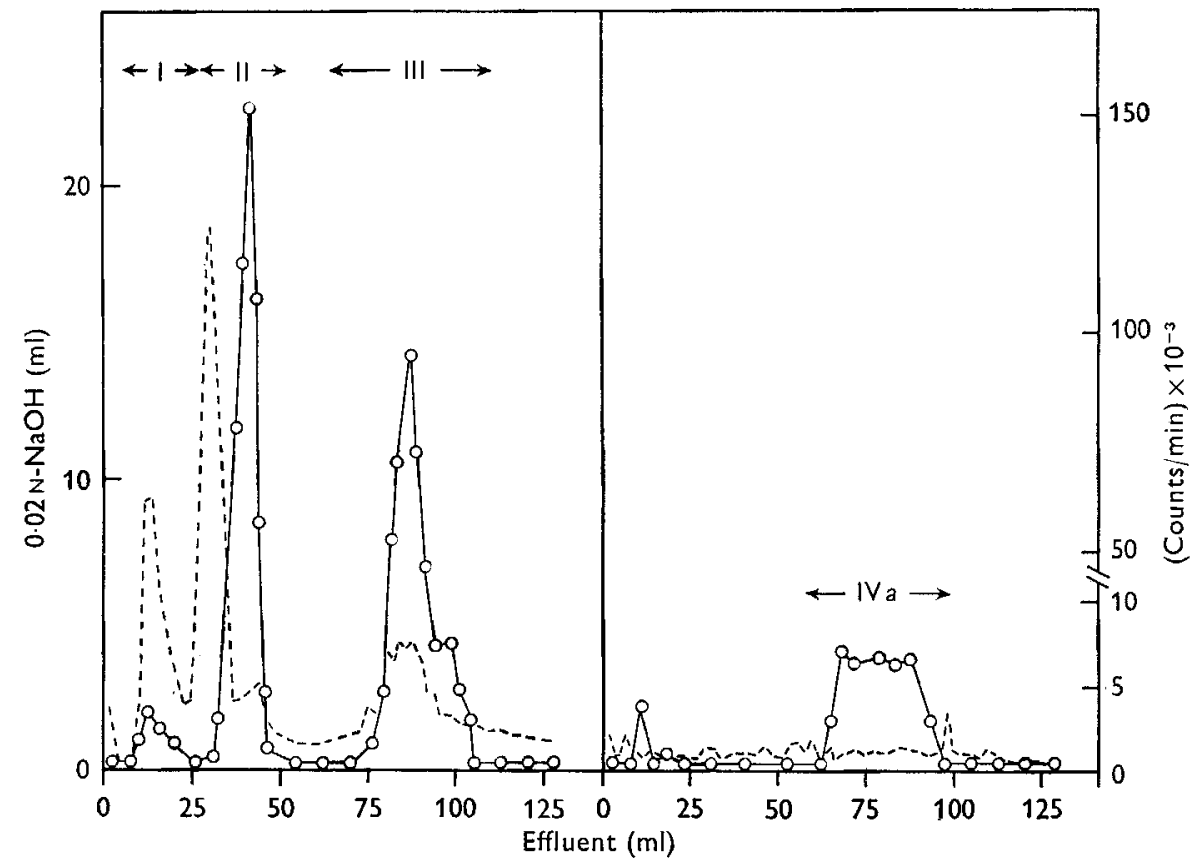

Fig. 2. Separation by chromatography of labelled metabolites from facces during 8 days after administration of $\left[24-{ }^{-14} \mathrm{C}\right]$ cholic acid to one rat reared on diet $\mathrm{D} 7$.

A-ether extract (phase system $\mathrm{F}_{\mathrm{I}}$ ); B-rechromatography of labelled compounds retained in the column after chromatography A (phase system F2). --., titration values; $\mathrm{O}-\mathrm{O}$, radioactivity.

$\begin{array}{ccccc}\begin{array}{c}\text { Phase } \\ \text { system }\end{array} & \text { Moving phase } & \mathrm{ml} & \text { Stationary phase } & \mathrm{ml} \\ \text { F I } & \text { Methanol:water } & 165: 135 & \text { Chloroform:heptan } & 45: 5 \\ \text { F2 } & \text { Methanol:water } & 130: 220 & \text { Chloroform:heptan } & 45: 5\end{array}$

Columns : $4 \mathrm{ml}$ stationary phase. Supported on $4.5 \mathrm{~g}$ hydrophobic Hyflo Super-Cel (JohnsManville, New York).

Fraction I. This fraction consisted of minor amounts of labelled compounds appearing with, or just after, the front. Subsequent TLC analysis showed that this fraction contained several unconjugated metabolites of cholic acid in addition to small amounts of unchanged cholic acid. 
Fraction 11 . This fraction was methylated and further fractionated on a column of aluminium oxide. Two labelled fractions were obtained (Fractions II $a$ and II $b$, Fig. 3). TLC showed that the major fraction (II $b$ ) consisted of methyl $3 \alpha$, I2 $\alpha$ dihydroxy-5 $\beta$-cholanate, and the minor fraction (II $a$ ) mainly of methyl $3 \beta$, г2 $\alpha$ dihydroxy $-5 \beta$-cholanate with trace amounts of two unidentified metabolites.

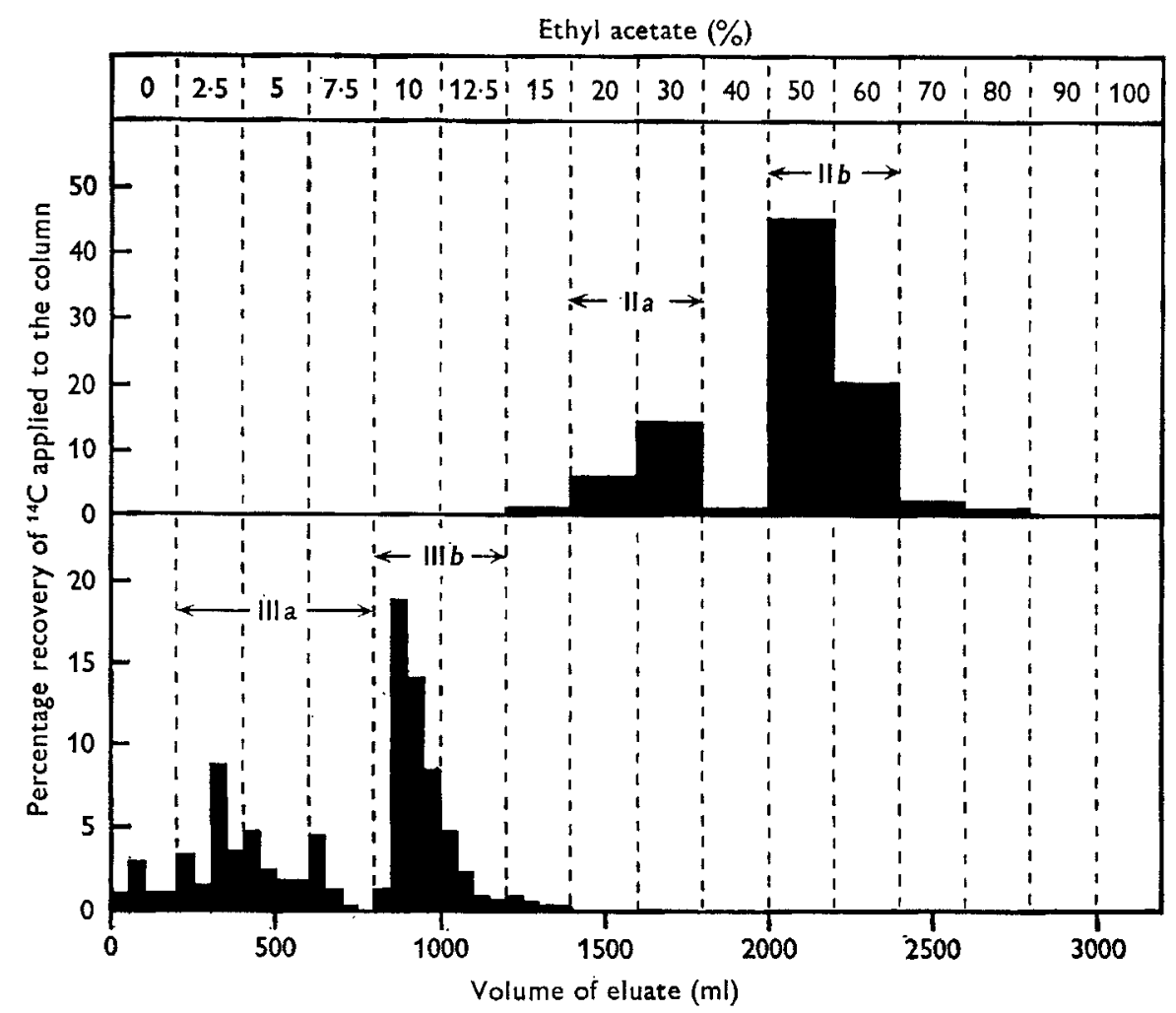

Fig. 3. Alumina fractionation of Fractions II and III (see Fig. 1). Column: $20 \mathrm{~g}$ aluminium oxide. The composition of the eluate is given as the percentage of ethyl acetate in benzene.

Fraction III. After methylation, this fraction was further separated on a column of aluminium oxide into two fractions (III $a$ and III $b$, Fig. 3). Subsequent TLC showed that Fraction III $b$ consisted of methyl $3 \alpha$-hydroxy-I2-keto- $5 \beta$-cholanate and that Fraction III $a$ consisted of four compounds: methyl $3 \beta$-hydroxy-I 2 -keto- $5 \beta$-cholanate, methyl $12 \alpha$-hydroxy-3-keto- $5 \beta$-cholanate, and two unidentified metabolites.

Fraction $I V$. This fraction represented the labelled material retained in the stationary phase after chromatography with phase system Fi. Rechromatography with phase system F 2 (Fig. 2) showed that only minor amounts of a metabolite were eluted at the position of 3, I2-diketo- 5 -cholanoic acid (Fraction IV $a$ ), but the TLC mobility of this metabolite revealed a difference between these two compounds. The major part of this fraction (IV $b$ ) remained in the stationary phase and consisted of saponifiable derivatives of labelled bile acids, which after hydrolysis (refluxing for $2 \mathrm{~h}$ in $\mathrm{N}-\mathrm{NaOH}$ 
in $90 \%$ methanol), and subsequent chromatography, were shown to be identical to the labelled bile acids found in Fractions I to IV.

\section{Labelled, faecal metabolites in rats receiving different diets}

Fig. 4 gives the percentage distribution of the labelled bile acid metabolites in Fractions I to IV, obtained after chromatography with phase system F I (Fig. I), of the ether extracts of faeces of rats in the different dietary groups. Comparison of the values for the percentage distribution of bile acids in the faeces of rats with and without prevention of coprophagy, showed that there were no significant differences between the six different animals in the same group, and so the means and ranges of these six combined values are given together in Fig. 4.

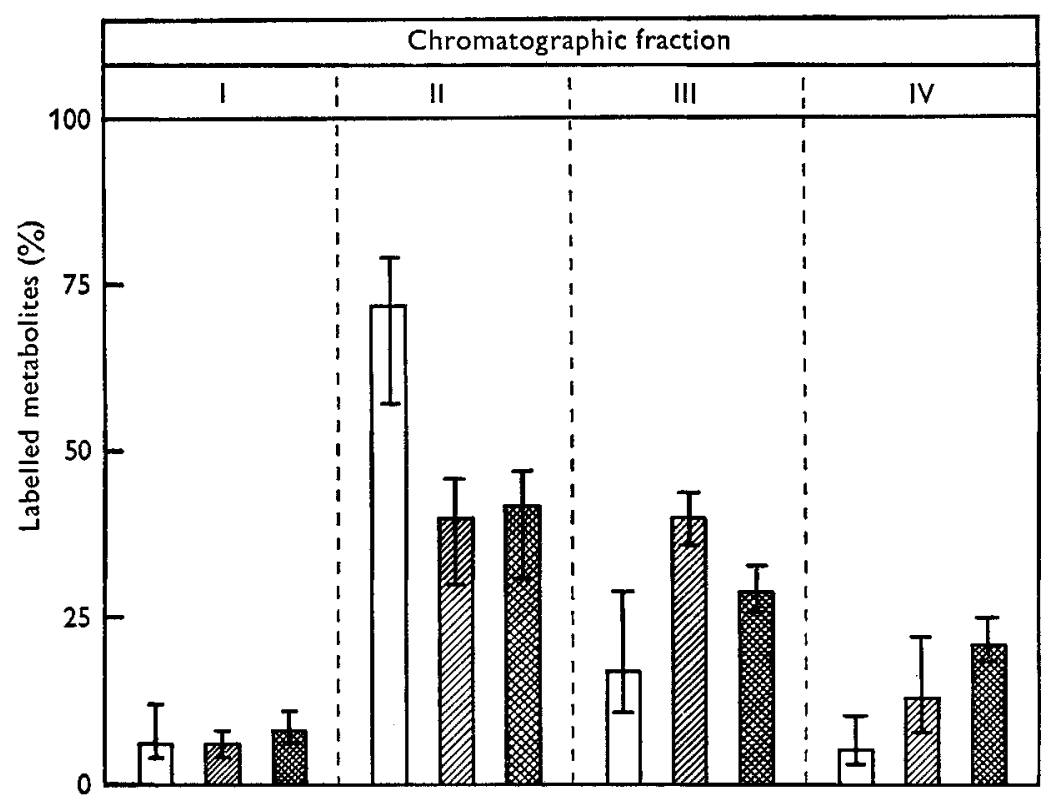

Fig. 4. Percentage distribution of labelled metabolites in Fractions I, II, III and IV (see Fig. I), obtained after chromatography of faecal bile acids from rats reared on diets $D_{7}, \square, D_{7}$ with cellulose, and pellets, Each bar represents the mean value for six rats and the vertical lines indicate the range.

Fraction I, which contained the unchanged cholic acid, contained only $6-8 \%$ of the labelled metabolites. The other labelled metabolites were recovered in Fractions II to IV, and thus lacked hydroxyl or keto groups at C-7. Fig. 4 also shows that the labelled bile acid metabolites from rats in the different dietary groups were distributed between the fractions in a similar manner, although, in rats receiving diet $D_{7}$, a greater proportion of metabolites was recovered in Fraction II. Approximately the same amount of labelled bile acids was recovered in Fractions II and III from rats receiving diet $\mathrm{D}_{7}$ with cellulose. In these rats and in those receiving pellets, a larger proportion of the dihydroxy-cholanoic acids (Fraction II) had been oxidized to monoketomonohydroxy-cholanoic acids (Fraction III) than in those receiving diet $\mathrm{D}_{7}$ alone. 
The labelled material in Fraction II was further analysed according to the scheme shown in Fig. I. The results, summarized in Table $\mathrm{I}$, indicate that more than $90 \%$ of the labelled material consisted of two metabolites with the TLC mobilities of $3 \alpha$, I2 $\alpha$-dihydroxy- $5 \beta$-cholanoic and $3 \beta$, 12 $\alpha$-dihydroxy- $5 \beta$-cholanoic acid. The ratio between these two isomers was roughly $3: 1$ in all the rats from the different dietary

Table I. Percentage distribution of labelled faecal metabolites of bile acids in Fraction II from groups of six rats reared on three different diets, $D_{7}, D_{7}+$ cellulose or pellets

\begin{tabular}{|c|c|c|c|c|c|c|}
\hline \multirow[b]{2}{*}{ TLC mobility of metabolite } & \multicolumn{2}{|c|}{$\mathrm{D}_{7}$} & \multicolumn{2}{|c|}{$\mathrm{D}_{7}+$ cellulose } & \multicolumn{2}{|c|}{ Pellets } \\
\hline & Mean & (Range) & Mean & (Range) & Mean & (Range) \\
\hline $\begin{array}{l}3^{\alpha}, 12 \alpha \text {-dihydroxy-5 } \beta \text {-cholanoic } \\
\text { acid }\end{array}$ & 73 & $(67-79)$ & 75 & $(7 I-8 I)$ & 74 & $(7 \mathrm{I}-80)$ \\
\hline $\begin{array}{l}3 \beta, \text { i2 } \alpha \text {-dihydroxy- } 5 \beta \text {-cholanoic } \\
\text { acid }\end{array}$ & 22 & $(14-28)$ & 18 & $(12-2,2)$ & 20 & $(15-23)$ \\
\hline Unknown metabolites & 5 & $(2-7)$ & 7 & $(3-9)$ & 6 & $(2-8)$ \\
\hline
\end{tabular}

Table 2. Percentage distribution of labelled faecal metabolites of bile acids in Fractions III and IV from rats reared on three different diets

(Mean values for the pooled faecal metabolites from six rats)

\begin{tabular}{|c|c|c|}
\hline Diet & $\begin{array}{c}\text { Metabolites with TLC } \\
\text { mobilities of } 3 \alpha \text {-hydroxy-12- } \\
\text { keto- } 5 \beta \text {-cholanoic acid and } \\
3 \beta \text {-hydroxy-1 } 2-\text { keto- } 5 \beta \\
\text { cholanoic acid in } \\
\text { Fraction III } \\
(\% \text { of labelled compounds } \\
\text { in Fraction III) }\end{array}$ & $\begin{array}{c}\text { Unknown metabolites } \\
\text { recovered in Fraction } \\
\text { IVa } \\
\text { (\% of labelled } \\
\text { compounds in } \\
\text { Fraction IV }\end{array}$ \\
\hline & 82 & 2 \\
\hline $\mathrm{D}_{7}+$ cellulos & 85 & 3 \\
\hline Pellets & 80 & 3 \\
\hline
\end{tabular}

groups. The percentage distribution of isotope between the other, unknown, metabolites in Fraction II was not determined. Fraction III, from each of the rats in the same dietary group, was combined, and the labelled material was analysed in the manner shown in the scheme in Fig. r. The results (Table 2) show that, irrespective of the diet received, Fraction III mainly consisted of metabolites with the TLC mobilities of $3 \alpha$-hydroxy-12-keto- $5 \beta$-cholanoic and $3 \beta$-hydroxy-12-keto- $5 \beta$-cholanoic acid. Only trace amounts of 12 -hydroxy-3-keto-cholanoic acid were found. Fraction IV from each of the rats in the same dietary group was also combined. The same small percentage amount of an unknown metabolite was recovered in Fraction IV $a$, from rats in all dietary groups (Table 2). Fraction IV, therefore, mainly consisted of saponifiable derivatives of labelled bile acids. 


\section{DISCUSSION}

In the present investigation the nature of the labelled faecal metabolites of $\left[24^{14} \mathrm{C}\right]$ cholic acid has been studied in rats on three different diets. The rats receiving the two semi-synthetic diets ( $D_{7}$ and $D_{7}$ with cellulose) gave a low excretion of bile acids, whereas this was high in rats receiving the commercial diet (Gustafsson \& Norman, 1969). The labelled faecal bile acids were excreted mainly in an unconjugated and $7 \alpha$-dehydroxylated form by rats in all three dietary groups. There was a lower excretion of monohydroxy-monoketo-cholanoic acids by rats fed on diet $D_{7}$ than by those fed on either of the other diets. No significant difference in the ratio of the $3^{\alpha-\text { and }} 3 \beta$-hydroxy isomers was observed in the faecal metabolites from rats in the different dietary groups. 3 , I2-diketo- $5 \beta$-cholanoic acid was not isolated from the faeces of any of the rats. The large amount of monoketo-mono-hydroxy-cholanoic acids excreted by rats on the commercial diet cannot be considered to be the main cause for the previously observed high excretion of bile acids by these rats (Gustafsson \& Norman, I969), since an even greater amount of these acids was formed in rats on diet $D_{7}$ with cellulose, whose faecal excretion of bile acids had been shown to be low.

It is not possible to state whether the increased formation of keto derivatives that was observed in rats fed on diet $\mathrm{D}_{7}$ with cellulose, or on the commercial diet, had been brought about by a change in the composition of the gastro-intestinal microflora, or by an influence of the diet on the activity of the micro-organisms that transformed the bile acids.

Fitzgerald, Gustafsson \& McDaniel (1964) have shown that, in rats where coprophagy was prevented with tail cups, there was a numerical change within certain bacterial groups in the faeces. In the present investigations, however, the bacteria were not enumerated, and the fact that the same composition of faecal bile acid metabolites was observed in animals with and without prevention of coprophagy seems to indicate that coprophagy prevention had not had any influence on that part of the intestinal microflora which is responsible for the transformation of bile acids.

In the rat, bile acids are mainly absorbed from the distal part of the ileum (Dietschy, Salomon \& Siperstein, I966), where transformations of bile acids by micro-organisms occur only to a minor extent (Norman \& Sjövall, I958). An extensive transformation of bile acids by microbial enzymes starts in the caecum, where various quantities of the metabolites formed are absorbed, and continues in the colon, before excretion in the faeces (Norman \& Sjövall, 1958). Various methods have been used for studying the extent of absorption of microbial metabolites of bile acids. The radioactive material in the portal blood has been analysed by means of reversed-phase-partition chromatography after the administration of tritium-labelled cholic acid to rats (Olivecrona \& Sjövall, I959), and radioactive bands have been found at the positions corresponding to the main cholic acid metabolites known to be formed in the caecum. Approximately $15 \%$ of the radioactive bile acids in the portal blood have been shown to be unconjugated, and deoxycholic acid formed approximately $50 \%$ of these unconjugated acids. It has also been found, in investigations using a gas chromatographic technique 
(Cronholm \& Sjövall, 1967), that the composition of the bile acids in the portal blood of rats could be influenced by the diet. Using two of the diets employed during the present investigation, they showed that the portal blood of rats fed on pellets contained more unconjugated bile acids $(36 \%)$ than that of rats receiving diet $D_{7}$ alone (10\%). Nevertheless, no significant differences were observed between the groups of rats on these two diets in the proportion of cholic and deoxycholic acid. However, the only microbial metabolite of cholic acid they determined was deoxycholic acid. Up to the present, there has been no report on the identification of the other cholic acid metabolites that may occur in portal blood. It is, therefore, not known whether the large amount of monoketo-hydroxy-cholanoic acids formed in the intestinal tract and appearing in the faeces, observed in the present investigation, may affect the composition of the bile acids in the enterohepatic circulation.

\section{REFERENCES}

Bollman, J. L., Cain, J. C. \& Grindlay, J. H. (1948). F. Lab. clin. Med. 33, I349.

Cronholm, T. \& Sjövall, J. (ז967). Eur. Y. Biochem. 2, 375.

Danielsson, H. (1963). Adv. Lipid Res. r, 335.

Danielsson, H., Eneroth, P., Hellström, K., Lindstedt, S. \& Sjövall, J. (I963). J. biol. Chem. $238,2299$.

Dawson, A. M. (1967). Gut 8, 1.

Dietschy, J. M., Salomon, H. S. \& Siperstein, M. D. (1966). F. clin. Invest. 45, 832.

Draser, B. S., Hill, M. J. \& Shiner, M. (1966). Lancet i, 1237.

Eneroth, P. (1963). F. Lipid Res. 4, I I.

Eneroth, P., Gordon, B., Ryhage, R. \& Sjövall, J. (1966). F. Lipid Res. 7, 511 .

Fitzgerald, R. J., Gustafsson, B. E. \& McDaniel, E. G. (1964). F. Nutr. 84, I 55.

Gall, S. L., Fenton, P. F. \& Cowgill, G. R. (I 948). F. Nutr. 35, I3.

Gustafsson, B. E., Midtvedt, T. \& Norman, A. (I966). F. exp. Med. 123, 413.

Gustafsson, B. E. \& Norman, A. (I968). Scand. $\mathscr{F}$. Gastroentrol. 3, 625.

Gustafsson, B. E. \& Norman, A. (I969). Br. F. Nutr. 23, 429.

Midtvedt, T. \& Norman, A. (1967). Acta path. microbiol. scand. 7x, 629.

Norman, A. (I964). Br. F. Nutr. 18, 173 .

Norman, A. \& Sjövall, J. (1958). F. biol. Chem. 233, 872 .

Olivecrona, T. \& Sjövall, J. (1959). Acta physiol. scand. 46, 284.

Tabaquchali, S. \& Booth, C. C. (1966). Lancet ii, 12. 